\title{
SYNTHESIS OF NEW WATER-SOLUBLE METAL-BINDING POLYMERS: COMBINATORIAL CHEMISTRY APPROACH
}

\author{
Mid-Year Progress Report \\ Barbara F. Smith \\ Los Alamos National Laboratory
}

June 1, 1997

Objectives: The first objective of this research is to develop rapid discovery and optimization approaches to new water-soluble chelating polymers. A byproduct of the development approach will be the new, selective, and efficient metal-binding agents. The second objective is to evaluate the concept of using water and organic soluble polymers as new "solid" supports for combinatorial synthesis.

\section{Technical Description of Work:}

Introduction: The technology under development, Polymer Filtration (PF), is a technique to selectively remove or recover hazardous and valuable metal ions and radionuclides from various dilute aqueous streams. Not only can this technology be used to remediate contaminated soils and solid surfaces and treat aqueous wastes, it can also be incorporated into facilities as a pollution prevention and waste minimization technology. Polymer Filtration uses water-soluble metalbinding polymers to sequester metal ions in dilute solution. The water-soluble polymers have a sufficiently large molecular size that they can be separated and concentrated using commercial ultrafiltration technology. Water, small organic molecules, and unbound metals pass freely through the ultrafiltration membrane while concentrating the metal-binding polymer. The polymers can then be reused by changing the solution conditions to release the metal ions. The metal-ions are recovered in concentrated form for recycle or disposal using a diafiltration process. The watersoluble polymer can be recycled for further aqueous-stream processing.

To advance Polymer Filtration technology to the selectivity levels required for DOE needs. fixture directions in Polymer Filtration must include rapid development, testing, and characterization of new metal-binding polymers. The development of new chelating molecules can be equated to the process of new drugs or new materials discovery. Thus, we want to build upon and adapt the combinatorial chemistry approaches developed for rapid molecule generation for the drug industry to the rapid development of new chelating polymers.

\section{Accomplishments to Date:}

The majority of the reported results is work performed at LANL (see Issues and Problems section). The three main areas that we have been focusing on are: (1) development of small ultrafiltration equipment compatible with organic-based combinatorial synthesis, (2) development of rapid, multiple assay techniques, and (3) development of polymer characterization techniques.

\section{(1) Small Ultrafiltration Equipment:}

One concept of this project is using soluble polymers as the "solid" support for the synthesis of the ligands. This is desirable because first we would directly obtain the target metal-binding 
ligands on the water-soluble polymer ready for assay, and secondly we would have the advantage of a homogenous synthesis as opposed to the heterogeneous resin synthesis. The ability to accomplish this could revolutionize the potential automatability of combinatorial synthesis because it is much easier to do accurate liquids addition and transfer than solid resins transfers. We are using water and organic soluble polyamine; the heteroatoms impart excellent aqueous solubility to the polymers. which is needed for processing, and they are also soluble in a number of organic solvents useful for organic synthesis. The advantages of these polymers is that they have greater capacity than the MeO-polyethyleneglycols used previously, as polyamines have multiple attachment points as opposed to only per strand and the separation is accomplished with ultrafiltration instead of precipitation.

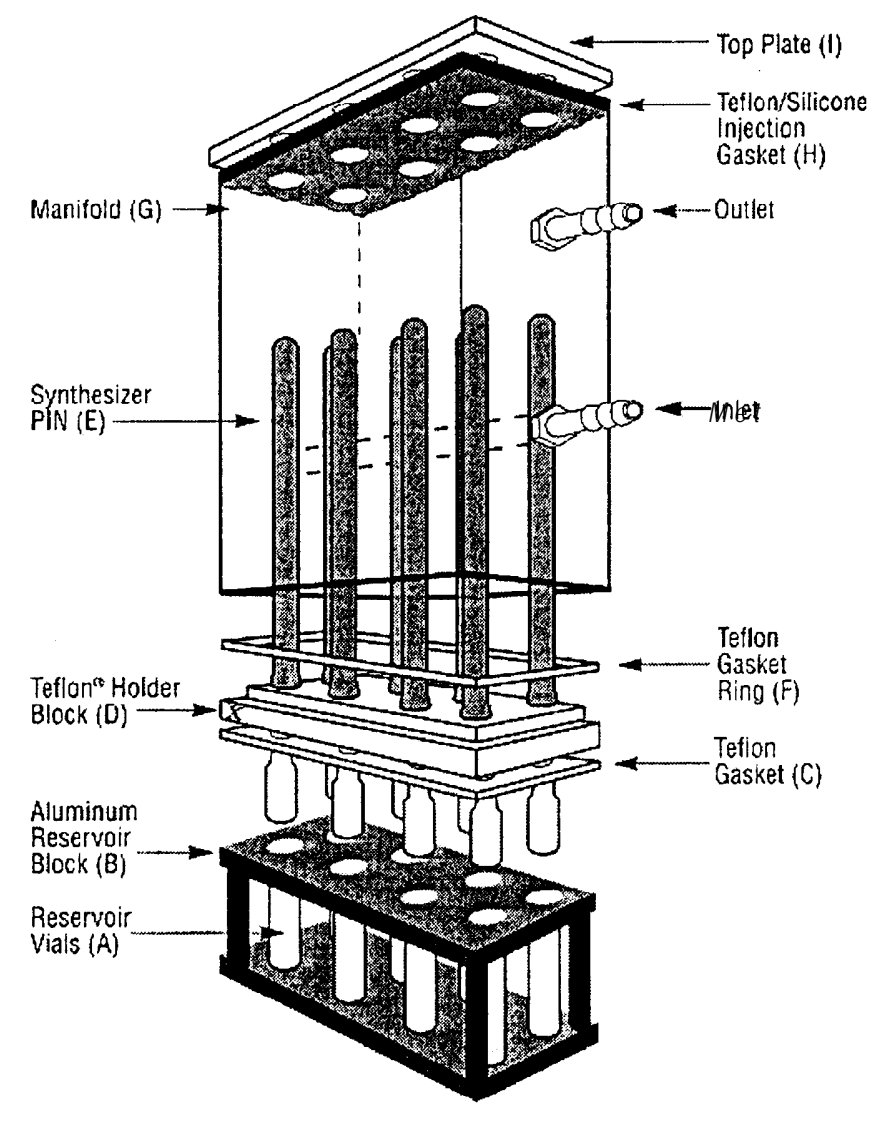

Figure 1. Schematic of Diversomer ${ }^{\mathrm{TM}}$ unit used for resin-based synthesis.

In order to accomplish our goal of using soluble polymers in a homogenous system it is necessary to develop an apparatus in which to perform parallel syntheses. The unit must be compatible with acids, bases, and organic solvents; it must be able to allow reactions to be performed under an inert, heating, or cooling atmosphere, and it has be allow for ultrafiltration for ready recovery of the soluble polymer. A commercially available unit produced by Diversomer ${ }^{\mathrm{TM}}$ that accomplishes this for resin synthesis is shown in Figure 1. The unit is an assembly of reaction vials held in an aluminum rack that can be heated or cooled if necessary. Into these vials are inserted glass fritted tubes into which the resin is placed. A cover is sealed with gaskets over the 
bottom assembly that allows for the addition of nitrogen for an inert atmosphere, and a top plate with an injection gasket through which the reagents can be added. At the completion of the reaction the fritted tubes, called pins, can be lifted from the reaction vials and the excess reagent is drained and rinsed from the resin. The full vials are replaced with empty vials and a second set of reactions can be performed on the same resin and the cycle repeated as necessary.

We are in the process of adapting this apparatus for ultrafiltration with soluble polymers as opposed to solid resins. Two approaches are being tested, the first where the polymer solution is placed on top of the membrane and we have dead-end filtration, the other is where the polymer solution is placed in a vial and the ultrafiltration unit is placed on top of the solution, or reverse dead-end filtration. The advantages of the second approach are that filtration is faster as we are not producing a gel concentration polarization layer on the membrane. The challenges we are having is materials compatibility. Many of the available membrane materials and holders are not compatible with the organic solvents we need to use. Thus, it has required finding alternate ways to perform the ultrafiltation. We are evaluating ceramic systems, all polysulfones systems, and disc inserts. Another variable to be optimized is the mode of force for ultrafiltration. We have used nitrogen pressure, centrifugal force, and vacuum to aid in the filtration.

(2) Polymer Assaying Techniques: The resulting chelating polymer developed from the combinatorial synthesis will be assayed for metal-ion binding strength and selectivity. Since there is the potential for preparing a large number of compounds, assaying techniques are needed that are quantitative, sensitive, rapid, multielement, nearly concurrent and require little sample. These techniques will need to be able to quantify metal ions in the presence of polymers.

We have chosen two techniques to develop first for the metal-binding studies: $\mathrm{x}$-ray fluorescence spectroscopy (XRF) and auto-radiography. These techniques were initially chosen as they appear to meet the requirements for rapid assay stated above.

$\underline{X-r a y}$ fluorescence spectroscopy (XRF): X-ray fluorescence (XRF) is considered a mature analytical technology usually regarded as a bulk analytical method with limited sensitivity and requiring several grams of sample for analysis. In the past, this had typically restricted application of XRF from small mass samples and to concentrations well above the parts-per-million level. With the advent of commercial, lab based x-ray microfluorescence instrumentation. a new tool to address these limitations of macro-XRF excitation instruments is now available. New developments in commercial instrumentation, software and sample support films provides analysts with sensitivities that approach and match those of graphite furnace atomic absorption and inductively coupled plasma-mass spectrometry.

Meltzer ( 1991) demonstrated that sub-ppm detection limits could be achieved on macroinstrumentation using a dried spot methodology. Dried spot methodology allows the direct analysis of radioactive samples using energy dispersive x-ray microfluorescence. Matching the excitation area of the x-ray spot to the dried sample spot, minimizes much of the scatter background and increases sensitivity. In addition, sensitivity is increased by removing the liquid matrix and concentrating on the dried residue components. Detection limits are demonstrated which are subppm in the liquid and approach less than $1 \mathrm{ng}$ in the dried spot.

We have been able to demonstrate the use of x-ray microfluorescence (XRMF) for trace analysis using the dried spot methodology for application to analysis of both RCRA and radioactive samples and wastes. The radioactive background is minimized by physically reducing the specimen size and 
permitting direct analysis of radioactive samples. We have also demonstrated that metal ions can be measured in the presence of water-soluble chelating polymers.

The procedure involves preparation of a polymer thin film as a sample support. Typical films are polycarbonate, polyester, and polypropylene that are 2-4 microns thick. The films are stretched and glued over a $35 \mathrm{~mm}$ slide mount. Normally 5-50 microliters of a liquid sample are applied to the surface of the film which is then allowed to dry. This analytical specimen is then analyzed directly. The liquid is placed on the well side of the slide, allowed to dry, and another layer of film is placed on top to seal the dried spot. Recent advances in thin film technology have led to the development of a commercially available sample system that can hold up to nine individual specimens on one slide, permitting multiple sample analysis and reducing both sample preparation time and cost per sample. An additional advantage of the commercial film is that it is 0.15 microns thick, which lowers the background and increases sensitivity. Our analyses were done using a Kevex (San Carlos, CA) Omicron x-ray microfluorescence spectrometer equipped with a rhodium $\mathrm{x}$-ray tube, operated at $50 \mathrm{kV}$ and $1 \mathrm{~mA}$ concentrations for each element. The AAPS proprietary software allows an almost universal calibration based on several known standards. Typical acquire times are around 300 live seconds. Spectrum processing includes escape peak identification, background modeling and subtraction and collection of both gross and net intensities.

We have been studying the variables in the analytical technique and they include the following:

SPOT SIZE VARIABLES:

- Solutions with metal concentration from $100 \mathrm{ppm}$ to $1 \mathrm{ppb}$ were studied for size variations. Air dried spots were around $0.25 \mathrm{~mm}$ in diameter for $1 \mathrm{ppm}$ solutions.

- Solutions with $1 \%$ polymer extraction solutions dried with spot sizes between 1.5 and 2.5 $\mathrm{mm}$ in diameter. Adhesion of the spots also appears to be a problem with the polymer solutions.

- Two pH values of 1.7 and 11.5 were examined and showed no effects on spot size

Temperature Effects:

- Liquid nitrogen cooled the sample too rapidly. A new approach to cooling needs to be determined.

- The film substrates appear to show no ill effects from temperature either cold or heat except wrinkles. The wrinkles may affect sensitivity due to changes in sample position and background scatter. This will be investigated further.

Drying Methods:

- Air drying gives the smallest spot sizes followed by desiccant. microwave, heating, heat with vacuum and vacuum-freeze dry (in order of increasing spot size). Microwave drying is the quickest, however spot sizes vary and we need to further test power levels and heating rates. This may be the best compromise in terms of speed of drying and compact spot development. Surface tension effects appear to be the biggest factor in determining the final size of the droplet.

- The fastest drying method was the heat with vacuum, although this along with the other vacuum approaches produces the largest and least concentrated spots. 
Film Thickness:

- The film substrate thickness has been measured experimentally with infrared spectrometry. This is to check manufacturer specifications. Film thickness variations could have significant impact on the sensitivity of the dried spot technique.

Auto-Radiography: Since all the target metal ions can be or are radionuclides, radiochemistry techniques can be used for rapid assaying of metal binding. The metal ions we are particularly interested in are plutonium $(\mathrm{Pu})$ and americium $(\mathrm{Am})$. The polymer/metal ion solution will be placed in a small well on an ultrafiltration membrane and filtered to near dryness. The sample spot is analyzed using a photographic film which is place in close proximity to the radiation source for a time sufficient to expose the film. The film is then developed in a developer solution, rinsed in water, and fixed in a fixer solution. In this way a whole series of wells will be spotted simultaneously using multiple syringe techniques, filtered simultaneously, and exposed to the photographic film simultaneously such that the density of the exposed film will allow determination of the best metal binders.

There are a number of parameters that are being evaluated to optimize this auto-radiographic approach. There are many possible film types that have various speeds. Currently we are evaluating small, dental x-ray film that is already prepackaged in a protective vinyl case. The issues of time of exposure, distance from radioisotope source, presence of a lead or fluorescence screen to enhance sensitivity, and development technique are under evaluation. Amercium-241 (Am-24 1) emits a 59.6 Kev gamma ray and plutonium-239 (Pu-239) emits a 5.10-5.16 Mev alpha particle. Because of their different half-lives, Am, 433 years, and Pu, 24,400 years, Am has many more decays/minute than $\mathrm{Pu}$, and consequently is a much hotter source for auto-radiographer than $\mathrm{Pu}$. Because their decay is different, the distances to the film, the amount of materials in the sample, back scattering, and enhancement issues all have to be considered.

Both of these analytical assay techniques are rapid, multielement, and the same membrane assay sheet could potentially be used in both methods. For example, if a plastic protective layer is used on the membrane, the membrane sheet can be placed in the XRF instrument and measured also. The two methods are complementary with the ability to assay both radio and non radoisotopes.

3) Polymer Characterization: Characterization of the polymeric lead materials pose especially unique and oftentimes complex problems owing to the fact that these materials are high in molecular weight and polydisperse, that is, not all of the molecules comprising a sample are either chemically or physically equivalent. Chemical derivatization and/or modification of polymers generally also introduces further significant changes in the properties of the materials. The design of polymers for specific functions and properties is obviously part of the molecular architecture of those molecules. To completely understand the properties of these materials it is highly desirable to correlate metal chelation with the molecular structure of the polymer.

Aqueous polymer behavior is in many respects much more difficult to characterize, predict, and evaluate than are organic soluble materials. This is in large part due to the complex nature of water itself, where ionic strength, added ions, $\mathrm{pH}$ and temperature all combine to form a very complex environment. Coupling this with the nature of polymeric chelating agents which are oftentimes ionic, the chemical and physical behavior of such systems can span a wide range. 
Understanding the behavior of polymers under such conditions will allow for the optimization of design and performance of these materials for their intended application.

Presently we are employing laser light scattering photometry to evaluate the change in hydrodynamic volume, association of polymers. weight-average molecular weight, and radius of gyration under conditions of varying $\mathrm{pH}$, temperature, ion strength, and added ions to understand the complex conformational aspects of these systems. Since we do not have the functionalized polymers to evaluate at this time (see Issues and concerns section), we are evaluating the polyamine backbone polymers to baseline the techniques and will determine the changes that occur upon functionalization. We have two different molecular weight polymers that we are evaluating, polyamine 1 is higher MW and polyamine- 2 is lower MW.

\section{Basic Principles of light scattering}

1) The amount of light scattered is directly proportional to the product of molecular weight and solute concentration.

2) The angular variation of scattered light is directly related to the size of the molecule.

\section{BASIC THEORY AND LIGHT SCATTERING EQUATIONS}

where:

$$
\mathrm{K}^{*} \mathrm{C} / \mathrm{R}(\theta)=1 / \mathrm{MP}(\theta)+2 \mathrm{~A}_{2} \mathrm{C}
$$

$\mathbf{K}^{*}=4 \pi^{2}(\mathrm{dn} / \mathrm{dc}) 2 \mathrm{n} 2 /\left(\mathrm{N}_{\mathrm{A}} \lambda^{4}\right)$, and $\mathrm{n}$ is the refractive index of the solvent.

$\mathbf{C}$ is the concentration of the solute molecules $(\mathrm{g} / \mathrm{ml})$.

$\mathbf{R}(\theta)$ is the fraction of light scattered relative to the incident beam.

$\mathbf{N}_{\mathrm{A}}$ is Avogadro's number.

$\lambda$ is the wavelength of light.

$\mathbf{d n} / \mathbf{d c}$ is the refractive index increment, which tells how much the refractive index of the solution varies with solute concentration.

$\mathbf{M}$ is the weight-average molecular weight of the solute.

A is the second virial coefficient (a measure of solvent-solute interaction).

$\mathbf{P}(\theta)$ is the "scattering function" which tells how the scattered light varies with angle. This variation is determined by $\left\langle\mathrm{r}^{2}\right\rangle$, the mean square radius (also known as radius of gyration). The bigger the $<$ $r^{2}>$, the bigger the angular variation.

$\left\langle\mathbf{r}^{2}\right\rangle$ is the mean square radius, describing the distribution of mass within the molecule, and it is given by the equation:

$$
<\mathrm{r}^{2}>=\sum \mathrm{r}_{\mathrm{i}}^{2} \mathrm{~m}_{\mathrm{i}} / \mathrm{M}
$$

The symbol $R(\theta)$, the Rayleigh ratio describes theangle-dependent light scattering, and it is described as

$$
R(\theta)=I_{\theta} r^{2} /\left(I_{0} V\right)
$$

where $I_{\theta}$ is the scattered intensity, $I_{0}$ is the intensity of the incident beam, $V$ is the volume of the scattered medium, and $\mathrm{r}$ is the distance between the scattering volume and the detector. Equation 3 implies that the dimensions of $\mathrm{R}(0)$ are length-', and the DAWN instruments are provided with 
vertically polarized light sources. When studying a solution of solvent plus solute, it is convenient to use R( $(\theta)$ represent the excess scattering of the solution compared to that of solvent alone as:

$$
R(\theta)=\left(I_{0}-I_{0 . \text { solvent }}\right) r^{2} /\left(I_{0} V\right) \quad \text { eq. } 4
$$

where $I_{0, \text { solvent }}$ is the scattered intensity of the solvent. Equation 3 is used for pure solvent and equation 4 is used for a solution. That means that we are merely describing the scattering after subtracting the "baseline" of pure solvent. In this case, $\mathrm{R}(8)$ is often called the excess Rayleigh ratio of the solute. The knowledge of Rayleigh ratio at a number of different angles leads directly to the weight-average molecular weight and mean square size of the solute molecules, malting $R(O)$ the most important measured quantity in light scattering.

The determination of molecular parameters of solute particles in solution is carried out by using the equation derived by Zimm, in which equation 1 is replaced by equation 5 .

$$
\mathrm{R}(\theta) / \mathrm{K}^{*} \mathrm{C}=\mathrm{Mw} \mathrm{P}(\theta)-2 \mathrm{~A}_{2} \mathrm{CM}_{\mathrm{w}}{ }^{2} \mathrm{P}^{2}(\theta)
$$

where $\mathrm{Mw}$ is the weight-average molecular weight, and other parameters defined previously. $\mathrm{P}(0)$ is related to $<\mathrm{r} 2>$ by the following equation:

$$
P(\theta)=1-2 \mu^{2}<r^{2}>/ 3+\ldots \quad \text { eq. } 6
$$

where $\mu=(4 \pi / \lambda) \sin (\theta / 2)$, and gives us the relation between $P(0)$ and mean square radius for any molecules regardless of molecular conformation in the limit of $\mu^{2}<\mathrm{r}^{2}><<1$. For a polydispersed sample. the mean square radius obtained is $\mathrm{z}$-average, usually written $\langle\mathrm{r} 2\rangle \mathrm{z}$. The determination of molecular weights and mean square radius are done by the use of Zimm, Debye or Berry plot. The Zimm and Debye plots are similar. To construct a Debye plot, we plot $y-=R(\theta) / K^{*} c \operatorname{VS~} \operatorname{Sin}^{2}(\theta / 2)+$ $\mathrm{kc}$ where $\mathrm{k}$ is a "stretch factor" which scales the contributions from $\mathrm{c}$ to be roughly equal to the contributions from $\sin 2(\theta / 2)$. It is chosen to be the reciprocal of the maximum concentration. In the case of the Zimm plot, we plot $y=K^{*} c / R(\theta) V S \operatorname{Sin}^{2}(\theta / 2)+k c$. The $y$-axis is the reciprocal of that used for Debye plot.

Molecular weight measurement by light scattering method

Measurement of dn/dc: was done with Wyatt Technology Optilab Differential refractometer 903 using a series of solutions of the polymer with different concentrations. This is a measurement of differential refractive index changes with concentrations.

The light scattering measurements: were done on DAWN F Laser photometer, using a series of concentrations of the polymer solution. The solutions were filtered with $0.2 \mu \mathrm{m}$ membrane filters to avoid light scattering from extraneous dust and other particles. The MWs were calculated from Zimm and Berry plots. 
MW of polyamine 1 in pure distilled water at pH 7.79: All results were calculated from Zimm, Debye and Berry plots.

\begin{tabular}{|l|l|l|l|}
\hline Serial \# & RMS $(\mathrm{nm})$ & A2 $(\mathrm{mol} \mathrm{ml} / \mathrm{g})$ & MW $(\mathrm{g} / \mathrm{mol})$ \\
\hline 1 & $142.5+/-7.8$ & $3.90+/-0.2 \mathrm{e}-04$ & $2.36+/-0.2 \mathrm{e}+5$ \\
\hline 2 & $127.4+/-6.2$ & $3.54+/-0.1 \mathrm{e}-04$ & $1.99+/-0.1 \mathrm{e}+5$ \\
\hline Average & $134.95+/-7.0$ & $3.72+/-0.15 \mathrm{e}-04$ & $2.175+/-0.15 \mathrm{e}+5$ \\
\hline
\end{tabular}

$\mathrm{DN} / \mathrm{DC}=0.174$

MW of polyamine 1 in $0.1 \mathrm{M} \mathrm{NaCl}$ at $\mathrm{p} \mathrm{H}$ adjusted to 7.0 , stock solution $=4.878 \mathrm{e}-03 \mathrm{~g} / \mathrm{ml}$.

\begin{tabular}{|l|l|l|l|}
\hline Serial \# & RMS $(\mathrm{nm})$ & $\mathrm{A} 2(\mathrm{~mol} \mathrm{ml} / \mathrm{g})$ & $\mathrm{MW}(\mathrm{g} / \mathrm{mol})$ \\
\hline 1 & $116.2+/-7.1$ & $8.49+/-0.9 \mathrm{e}-05$ & $5.18+/-0.4 \mathrm{e}+5$ \\
\hline 2 & $129.2+/-8.5$ & $6.41+/-0.6 \mathrm{e}-05$ & $6.31+/-0.5 \mathrm{e}-+5$ \\
\hline 3 & $106.5+/-7.2$ & $9.48+/-1.0 \mathrm{e}-05$ & $4.67+/-0.3 \mathrm{e}+5$ \\
\hline Average & $117.3+/-7.6$ & $8.13+/-0.83 \mathrm{e}-05$ & $5.39+/-0.4 \mathrm{e}+5$ \\
\hline
\end{tabular}

MW of polyamine-2 in $0.1 \mathrm{M} \mathrm{NaCl}$ at $\mathrm{p} \mathrm{H}$ adjusted to 7.0 , stock solution $=8.036 \mathrm{e}-03 \mathrm{~g} / \mathrm{ml}$.

\begin{tabular}{|l|l|l|l|}
\hline SERIAL\# & RMS $(\mathrm{nm})$ & A2 $(\mathrm{mol} \mathrm{ml} / \mathrm{g})$ & MW $(\mathrm{g} / \mathrm{mol})$ \\
\hline 1 & $113.0+/-7.5$ & $1.69+/-0.2 \mathrm{e}-04$ & $1.49+/-0.1 \mathrm{e}+05$ \\
\hline 2 & $106.0+/-7.2$ & $-1.37+/-0.5 \mathrm{e}-04$ & $1.42+/-0.1 \mathrm{e}+05$ \\
\hline 3 & $97.4+/-7.0$ & $-1.2+/-0.5 \mathrm{e}-04$ & $1.30+/-0.1 \mathrm{e}+05$ \\
\hline 4 & $109.1+/-6.0$ & $2.02+/-0.2 \mathrm{e}-04$ & $1.41+/-0.08+05$ \\
\hline Average & $106.38+/-6.9$ & $1.57+/-0.3 \mathrm{e}-04$ & $1.405+/-.095 \mathrm{e}+05$ \\
\hline
\end{tabular}

MW of polyamine 2 in $1 \mathrm{M} \mathrm{NaCl}$ at $\mathrm{pH}$ adjusted to 7.0 , stock solution $=8.108 \mathrm{e}-03 \mathrm{~g} / \mathrm{ml}$

\begin{tabular}{|l|l|l|l|}
\hline Serial\# & RMS $(\mathrm{nm})$ & A2 $(\mathrm{mol} \mathrm{ml} / \mathrm{g})$ & MW $(\mathrm{g} / \mathrm{mol})$ \\
\hline 1 & $106.9+/-7.6$ & $2.62+/-1.0 \mathrm{e}-04$ & $1.48+/-0.1 \mathrm{e}+05$ \\
\hline 2 & $116.8+/-7.7$ & $2.39+/-1.0 \mathrm{e}-04$ & $1.65+/-0.1 \mathrm{e}+05$ \\
\hline 3 & $109.0+/-7.3$ & $1.60+/-0.3 \mathrm{e}-04$ & $1.36+/-0.1 \mathrm{e}+05$ \\
\hline 4 & $129.4+/-15.8$ & $1.62+/-0.3 \mathrm{e}-04$ & $1.44+/-0.3 \mathrm{e}+5$ \\
\hline Average & $115.53+/-9.6$ & $2.06+/-0.65 \mathrm{e}-04$ & $1.48+/-0.83 \mathrm{e}+5$ \\
\hline
\end{tabular}

MW of polyamine- 2 in $5 \mathrm{M} \mathrm{NaCl}$ at $\mathrm{pH}$ adjusted to 7.0 , stock solution $=8.036 \mathrm{e}-03 \mathrm{~g} / \mathrm{ml}$.

\begin{tabular}{|l|l|l|l|}
\hline Serial \# & RMS $(\mathrm{nm})$ & A2 $(\mathrm{mol} \mathrm{ml} / \mathrm{g})$ & $\mathrm{MW}(\mathrm{g} / \mathrm{mol})$ \\
\hline 1 & $132.4+/-7.8$ & $3.07+/-0.2 \mathrm{e}-04$ & $1.24+/-0.09 \mathrm{e}+05$ \\
\hline 2 & $117.4+/-8.5$ & $3.32+/-0.2 \mathrm{e}-04$ & $1.06+/ 00.09 \mathrm{e}+5$ \\
\hline 3 & $106.9+/-8.3$ & $3.56+/-0.2 \mathrm{e}-04$ & $9.46+/-0.8 \mathrm{e}+04$ \\
\hline 4 & $128.0+/-9.8$ & $3.18+/-0.2 \mathrm{e}-04$ & $1.16+/-0.1 \mathrm{e}+05$ \\
\hline Average & $121.2+/-8.5$ & $3.28+/-0.2 \mathrm{e}-04$ & $1.102+/-0.1 \mathrm{e}+05$ \\
\hline
\end{tabular}


MW of polyamine-2 in $1 \mathrm{M} \mathrm{NaCl}$ at $\mathrm{pH} 3.3$, Stock solution $=7.00 \mathrm{e}-03 \mathrm{~g} / \mathrm{ml}$. MW of polyamine-2 in $1 \mathrm{M} \mathrm{NaCl}$ at $\mathrm{pH} 10$, Stock solution $=4.108 \mathrm{e}-03 \mathrm{~g} / \mathrm{ml}$

\begin{tabular}{|l|l|l|l|}
\hline $\mathrm{pH}$ & RMS(nm) & A2 $(\mathrm{mol} \mathrm{ml} / \mathrm{g})$ & $\mathrm{MW}(\mathrm{g} / \mathrm{mol})$ \\
\hline 3.3 & $68.8+/-6.5$ & $1.66+/-0.3 \mathrm{e}-04$ & $1.14+/-0.1 \mathrm{e}+05$ \\
\hline 7.0 & $99.3+/-7.3$ & $1.74+/-0.3 \mathrm{e}-05$ & $1.23+/-0.09 \mathrm{e}+05$ \\
\hline 10.0 & $69.6+/-6.4$ & $2.4+/-1.0 \mathrm{e}-04$ & $1.24+/-0.1 \mathrm{e}+05$ \\
\hline
\end{tabular}

We can see from these results that the hydrodynamic volume and molecular weight is greatly effected by $\mathrm{pH}$, and salt concentration. We will followup on the evaluation of the functionalized polymers. The next area of evaluation will be to look at the NMR of these materials.

\section{Projections:}

We will not complete the scope for FY97 in FY97, but will carry the work over into FY98.

There is considerable work to complete in FY97, but because our university collaborators have only received their funding at the end of May, we will be behind schedule. Almost all the tasks that were to be performed by our university collaborators needs to be completed. This includes much of the methods development for the synthesis, and characterization of the polymers.

\section{Funding:}

\$224.7K allocated thus far Nov. \$16.7K, Dec \$17.9K, Jan 21.4, Feb \$5.9K, March \$22.3K, April $\$ 6.6 \mathrm{~K}$ for total $\$ 91.6 \mathrm{~K}(40.7 \%$ spent $)$

University collaborators have $\$ 0 \mathrm{~K}$ spending; only received funding at end of May.

We project about $\$ 40 \mathrm{~K}$ will be spent for the rest of the year unless our University collaborators can rapidly come up to speed.

The project will be accomplished within the budget allocated.

\section{Issues/Problems:}

The has been a major impediment to this project and that is that we are depending upon our university collaborators for much of the synthetic methods development and they only received their funding at the end of May. It will not necessarily change the scope, put it changes the time table, as we have slowed our work to get in step with the collaborators. All is required is that the time table be changed to match the allocation time of our collaborators. This means that we be have to be allowed to carry over some of the funds from this fiscal year to next to get back on schedule.

\section{Corrective Actions:}

We have held back on our work efforts to try to be in sink with our university collaborators. We intend on carrying over the funds to next year, keep the same scope, but increase the schedule. 